ORIGINAL ARTICLE

AFRICAN JOURNAL OF CLINICAL AND EXPERIMENTAL MICROBIOLOGY SEPTEMBER 2013 ISBN 1595-689X VOL14 No.3 AJCEM/1322

COPYRIGHT 2013

http://www.ajol.info/journals/ajcem

AFR. J. CLN. EXPER. MICROBIOL. 14(3): 180-183. http:/ / dx.doi.org/10.4314/ajcem.v14i3.11

\title{
PRESUMPTIVE DIAGNOSIS OF SCHISTOSOMA HAEMATOBIUM AND SCHISTOSOMA MANSONI USING MICROSCOPY AS GOLD STANDARD IN A RIVERRINE COMMUNITY OF SOUTHWESTERN NIGERIA
}

\author{
NASSAR A S (1), ADETORO T A ${ }^{(1)}$, ADEBIMPE W A ${ }^{(2)}$ and MUHIBI M A ${ }^{(3)}$ \\ (1)Biomedical Science Department, Ladoke Akintola University of Technology, Ogbomoso, (2) Department of Community \\ Medicine, College of Health Sciences, Osun State University, Osogbo, $\left({ }^{3}\right.$ Haematology Department, Ladoke Akintola University \\ of Technology Teaching Hospital, Osogbo.
}

Correspondence- muhibudeen@yahoo.com

\begin{abstract}
A cross-sectional study was carried out in Ilie community of Olorunda Local Government Area in Osun state, southwestern Nigeria to comparatively evaluate the presumptive diagnosis of schistosoma infections using microscopy as gold standard. One hundred and thirty seven consented primary school children aged 4 to 15 years were examined for presence of schistosome eggs. The urine samples were analyzed with urinalysis strips for microhaematuria as indicators of presumptive diagnosis for urinary schistosomiasis while fecal samples were analyzed with fecal occult blood test kits for occult blood detection as an indicator of presumptive diagnosis for intestinal schistosomiasis. The indicators of presumptive diagnosis were compared with microscopy examination of urine and stool while sensitivity and specificity of the presumptive diagnostic methods were determined. The results of the prevalence showed that $107(78.1 \%)$ had co- infection and overall prevalence of $73.5 \%$ and $26.3 \%$ recorded for both S. haematobium and $S$ mansoni infection respectively. It was observed that the use of microhaematuria alone had $52 \%$ sensitivity and $91.67 \%$ specificity while stool occult blood recorded 73.685 and $66.67 \%$ for sensitivity and specificity respectively. This study shows that presumptive diagnosis of urinary schistosomiasis is significantly more sensitive $(P<0.05)$ than intestinal schistosomiasis. Also, various degrees of co- infections were observed across all age groups of study subjects with age group 1012years exhibiting highest co- infection rate 48(13.4); and tendency towards increased transmission and re-infection. Use of these alternatives is recommended in resource limited settings, to be confirmed by gold standard when feasible.
\end{abstract}

Keywords: Presumptive diagnosis, Schistoma haematobium, Schistoma mansoni, Microscopy, Holoendemic Community.

\section{INTRODUCTION}

Schistosomiasis is one of the most prevalent parasitic infection after malaria, with nearly 207 million people infected, and 779 million currently at risk in 76 countries of the tropics where the disease is endemic (1).In sub- Sahara Africa, about 192 million are found to be infected with the disease (2).It is the most prevalent of the waterborne parasitic diseases and one of the greatest risks to health in rural areas of the developing world and the intensity of the infection rises with age and peaks usually between 15and 20 years of age (3). Schistosomiasis is a parasitic disease caused by blood flukes (Trematode). Intestinal Schistosomiasis caused by $S$ mansoni occurs in 52 nations including Caribbean countries (4) while $S$ haematobium,- causative agent of urinary schistosomiasis- is endemic in 54 countries mainly in Africa and Eastern Mediterranea (5).Co-Infection of S.haematobium and S.mansoni has been reported in various parts of Africa and Nigeria (6,7and 8). Most studies on the epidemiology of schistosomiasis are usually based on microscopic parasitological technique which is often cumbersome, time consuming and reagent dependent (9) .The resources to accomplish this is often absent in rural areas of
Nigeria. The lack of a widely accepted alternative diagnostic technique in these areas, are responsible for the inadequate containment of the continued transmission of these infections. Thus, this study is directed at establishing presumptive diagnostic methods and comparing same with microscopy as gold standard.

\section{METHODOLOGY}

Study Area: The study was carried out in Ilie community of Osun State. Ilie is situated in Olorunda Local Government Area of Osun State, Southwestern Nigeria. Its geographical coordinates are $7^{\circ} 58^{\prime} 0^{\prime \prime}$ North, $4^{\circ} 32^{\prime} 0^{\prime \prime}$. The community has a population less than 5000 persons with an annual rate growth of about 3\%. The inhabitants are of the ethnic Yoruba speaking group with farming and fishing as their predominant occupation. In the community, there are two predominant primary schools with one secondary school. More than $70 \%$ of the population of the community access water from the local river for use. 
Sampling Technique

A total of 137 subjects within the age range of 4-15 years were randomly selected among the primary and junior secondary schools in the study area. At least, 10 eligible subjects were selected in each class until maximum sample size was reached. Before the commencement of sample collection and questionnaire administration, Ethical approval were obtained from both Osun State Universal Basic Education Board and Local inspector of Education while informed consents were obtained from the parents/guardians through Parents Teachers Association(PTA) forum. A Pre-survey visit was made to the school in order to familiarize and educate the school authorities about the importance of the study.

\section{Sample Collection and Processing}

Two specimen containers were given to each subject and the procedure for introduction of stool and urine specimens into the containers was carefully explained to them .Stool and urine samples were labeled before processing. The urine samples were analyzed within 6 hours of collection to prevent hatching of the schistosome eggs. The urine specimens were observed for presence of colour, visible blood and turbidity. Microhaematuria and proteinuria analysis were carried out by using urinalysis strip (combistic 9) Urine deposit obtained by centrifuging $10 \mathrm{ml}$ of urine in a conical tube $1000 \mathrm{~g}$ was examined microscopically using 10x objective with condenser closed sufficiently to give good contrast. Stool analysis, and faecal occult blood test were carried out as described by Chessbrough (2005) (9). Data obtained from stool and urine analysis were used to evaluate their sensitivity and specificity according to World Health Organisation Format (10) using microscopy as Gold standard.

Sensitivity $(\%)=$ Total true positive $x 100 /$ Total true positive + Total false positive

Specificity $(\%)=$ Total true negative $x 100 /$ Total true negative+Total false positive

\section{RESULTS}

From a total of 137 examined, 107(78.1\%) were found to have co- infection and the prevalence did not show significant variation with age $(\mathrm{P}<0.05)$. An overall prevalence of $73.5 \%$ urinary schistosomiasis and $26.3 \%$ of intestinal schistosomiasis was observed in this study (Table 1).

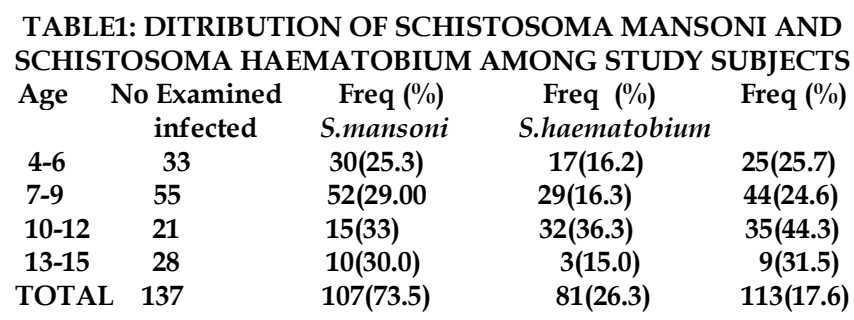

Using strip microhaematuria test alone as an indicator of presumptive diagnosis of urinary schistosomiasis in this study, it was observed that there was 38.2\% true positive results, $35.3 \%$ false negative results, $2.2 \%$ false positive results and $24.3 \%$ true negative results (Table2).

\begin{tabular}{|c|c|c|}
\hline \multicolumn{3}{|c|}{$\begin{array}{l}\text { MICROHAEMATURIA TECHNIQUE FOR } \\
\text { SCHISTOSOMA HAEMATOBIUM USING } \\
\text { MICROSCOPY AS GOLD STANARD }\end{array}$} \\
\hline $\begin{array}{r}N \\
\text { haeı }\end{array}$ & $\begin{array}{l}\text { ro - } \\
\text { turia(0) }\end{array}$ & Microscopy(\%) \\
\hline No of true Positive(\%) & 38.2 & 50.3 \\
\hline False Negative $(\%)$ & 35.3 & 62.3 \\
\hline False Positive( $\%$ ) & 2.2 & 20.3 \\
\hline True Negative $(\%)$ & 24.3 & 13.4 \\
\hline Sensitivity(\%) & 52.0 & \\
\hline Specificity(\%) & 91.67 & \\
\hline
\end{tabular}

TABLE 3: SENSITIVITY AND SPECIFICITY OF OCCULT BLOOD TECHNIQUE FOR SCHISTOSOMA MANSONI AND MICROSCOPY GOLD STANARD Occult Blood(\%) Microscopy(\%)

$\begin{array}{lcc}\text { No of true Positive(\%) } & 14.80 & 26.30 \\ \text { False Negative(\%) } & 53.20 & 62.30 \\ \text { False Positive }(\%) & 26.60 & 12.20 \\ \text { True Negative(\%) } & 62.70 & 76.30 \\ \text { Sensitivity(\%) } & 73.68 & \\ \text { Specificity }(\%) & 66.67 & \end{array}$

Table 4: DISTRIBUTON OF CO-INFECTION AMONG AGE -GROUPS

Co-Infecton Age(years) Freq (\%) Infected

A.lumbricoide+

S.haematobium

S.haematobium+

S.mansoni

4-9

39(17.40)

A.lumbricoidest

S.mansoni

10-12

48(34.40)

S.mansoni +

A.duodenale

12-13

38(11.70)

13-15

12(4.30) 
This indicator thus has 52\% sensitivity, 91.67\% specificity, $94.55 \%$ positive predictive value and $40.74 \%$ negative predictive value. Considering sensitivity and specificity of stool occult blood technique with microscopy for the diagnosis Schistosoma mansoni, it was observed that this indicator has $14.89 \%$ true positive results, $5.32 \%$ false negative results, $26.60 \%$ false positive results and $53.19 \%$ true negative results(Table3). This indicator thus has $73.68 \%$ sensitivity and $66.67 \%$ specificity with a positive predictive value of $35.90 \%$ and a negative predictive value of $90.9 \%$.the co-infection observed include Ascaris lumbricoides and Schistosoma haematobium, Schistosoma haematobium and Schistosoma mansoni, Ascaris lumbricoides and Schistosoma mansoni, Schistosoma mansoni and hook worms and highest co infection observed among the age group10-12 years.

\section{DISCUSSION}

This research work revealed the possibility of the use of presumptive diagnosis in rural communities that lack the facilities of microscopy. This study agrees with similar studies in endemic areas (8). High prevalence of Schistosomiasis recorded suggests endemicity and probably leads to higher degree of environmental contamination which as a result of water contacts activities; promotes the infection rate. S. hamatobium are more common than S.mansoni infection and this finding agrees with studies carried out by Gundiri and Okwuosa (2001) which reported that urinary schistosomiasis is more prevalent than intestinal schistosomiasis (10).

Using micro haematuria as indicators of presumptive diagnosis, for urinary schistosomiasis with

\section{REFERENCES}

1. Steinmann P., Keiser J., Bos R., Tanner M. and Utzinger J. (2006). Schistosomiasis and water resources development: systematic review meta-an nalysis and estimates of people at risk. The Lancet Infectious Diseases, 6(7): 411-425.

2. WHO (1990). Health Education and control of schistosomiasis. World Health Organization manual. Geneva 280pp.

3. World Health Organization (2010). Schistosomiasis Fact Sheet No 115 February, 2010; Available at http://www.who.int/mediacentre/facesheets/fs115 /en/index.html. Accessed Oct 5, 2010

4. Farid, A. W., Gamal, E., Yoser, E. B., Imam, R., Eman, M. and Thomas, S. (2000). The epidemiology of schistsomaisis in Egypt: Methods, Training and quality control of Clinical and ultrasound examinations. American Journal of Tropical Medicine and Hygiene, 62 (2) 5:17-20.

5. Sovionli, A., Bilndly, L. and Thomos, A. (1992); instestinal parasitic infections, a public health microscopy as the gold standard, the indicator is not reliable enough for presumptive diagnosis. High true positive results recorded may probably due to other health conditions and subjects into further investigation hence microscopy which is the gold standard remains the choice of examination of urinary schistosomiasis. Fecal occult blood for intestinal schistosomiasis reveals $73.68 \%$ sensitivity and $66.67 \%$ specificity, thus, making it a good alternative for investigation of intestinal schistosomiasis in an area where microscopy method is absent. This finding agrees with the results of previous studies around the globe (11,12 and 13) and in Nigeria (14, 15, 16, 17 and 18). Other parasites discovered in this course of study are Ascaris lumbricoides and hookworm. This indicates that more research needs to be done on the prevalence of helminthic infections in this community

In conclusion, more investigations need to be done on the use of microhaematuria and proteinuria as indicators of presumptive diagnosis for urinary schistosomiasis in other endemic areas in Nigeria because most places lack adequate facilities for the use of microscopy method. Also, adequate interventional strategies should be put in place at the endemic regions to prevent transmission and reinfection. This should be done with adequate information of the etiological and disease transmission knowledge of schistosomiasis to local inhabitants of endemic areas. Deployment of more human and material resources to endemic communities will help in stemming the spread of both urinary and intestinal schistosomiasis.

problem. American journal of Tropical medicine and Hygiene 43: 285 - 298.

6. Vennervald, B.J. and Dunne, D.W. (2004). Morbidity in schistosomiasis: an update. Curr Opin Infect Dis. 17(5):439-47.

7. Mwanakasale, V., Siziya, S., Mwansa, J., Koukounari, A. and Fenwick, A. (2009). Impact of iron supplementation on schistosomiasis control in Zambian school children in a highly endemic area. Malawi Med J. 21(1):12-8.

8. Nassar, A.S. and Abdulazeez, A. A.(2007). Presumptive diagnosis of intestinal schistosomiasis using kato-katz as gold standard reference technique. Journal of life and environmental sciences. Vol 9 (1) $508-512$

9. Cheesbrough, M., (2005). District Laboratory Practice in Tropical Countries. Part 1 Second Edition. 
10. Gundiri, M.A and Okwuosa, V.N (2000) An environmental study of Schistosomiasis and other parasitic infections in Langtang, Plateau state,Nigeria.Nig.J.parastol.2.Absracts.24th

Annualconference 9;15

11. Farid, A. W., Gamal, E., Yoser, E. B., Imam, R., Eman, M. and Thomas, S. (2000). The epidemiology of schistsomaisis in Egypt: Methods, Training and quality control of Clinical and ultrasound examinations. American Journal of Tropical Medicine and Hygiene, 62 (2) 5:17-20.

12. Eric, M. M., John, H. O. and Charles, H. K. (1996). Dynamics and control of Schistosoma haematobium transmission in Kenya: An overview of the Msambeni project. American Journal of TropicalMedicine and Hygiene, 55 (5):127-134.

13. Kabatereine, N. B., Brooker, S., Tukahebwa, E. M., Kazibwe, F. and Onapa, A. W. (2004). Epidemiology and geography of Schistosoma mansoni in Uganda: implications for planning control. Tropical Medicine and International Health, 9(3):372-80
14. Ramsay, G. W. (1935). A study of schistosomiasis and certain helminthes infections in Northern Nigeria. British Medical Journal,8: 20-10.

15. Iarotski,I.A and Davis, A.(19810.The Schistosomiasis transmission at high altitudecrater lakes in Western Uganda.BMC InfectDis.8;10

16. Ofoezie,I.E,Bolton,P.,Imevbore,A.M.A and Christensen, N.O(2002) Schistosomiasis and other heminth infection in irrigation scheme in Sokoto,Katsina AND Kebbi states of Nigeria.The Nigeria Journal of Parasitology,17;31-37.

17 Anosike, J. C., Okafor, F. C. and Onwuliri, C. O. E. (1992). Urinary schistosomiasis in Toro Local Government Area of Bauchi State, Nigeria. Helminthologia, 29: 177-179.

18. Anyanwu, G. I. and Okoro, O. C. (2002). Observation of urinary schistosomiasis in school children in Jos, Plateau State, Nigeria. International. Journal of Environmental Health and HumanDevelopment, 3(1): 31-34. 